RM3-TH/11-17

CERN-PH-TH/2011-298

\title{
The Mystery of Neutrino Mixings
}

\author{
Guido Altarelli \\ Dipartimento di Fisica, Universita' di Roma Tre \\ Rome, Italy \\ and \\ CERN, Department of Physics, Theory Division \\ CH-1211 Genève 23, Switzerland \\ E-mail: guido.altarelli@cern.ch
}

\begin{abstract}
In the last years we have learnt a lot about neutrino masses and mixings. Neutrinos are not all massless but their masses are very small. Probably masses are small because neutrinos are Majorana particles with masses inversely proportional to the large scale $\mathrm{M}$ of lepton number $(\mathrm{L})$ violation, which turns out to be compatible with the GUT scale. We have understood that there is no contradiction between large neutrino mixings and small quark mixings, even in the context of GUTs and that neutrino masses fit well in the SUSY GUT picture. Out of equilibrium decays with $\mathrm{CP}$ and $\mathrm{L}$ violation of heavy $\mathrm{RH}$ neutrinos can produce a B-L asymmetry, then converted near the weak scale by instantons into an amount of B asymmetry compatible with observations (baryogenesis via leptogenesis). It appears that active neutrinos are not a significant component of Dark Matter in the Universe. A long list of models have been formulated over the years to understand neutrino masses and mixings. With the continuous improvement of the data most of the models have been discarded by experiment. The surviving models still span a wide range going from a maximum of symmetry, with discrete non-abelian flavour groups, to the opposite extreme of anarchy.
\end{abstract}

\section{Introduction}

Experiments on neutrino oscillations, which measure differences of squared masses as well as mixing angles 1, 2) have established that neutrinos have a mass. Two distinct oscillation frequencies have been first measured in solar and atmospheric neutrino oscillations and later confirmed by experiments on earth, like KamLAND, K2K and MINOS. Two well separated differences need at least three different neutrino 
mass eigenstates involved in oscillations so that the three known neutrino species can be sufficient. Then at least two $\nu$ 's must be massive while, in principle, the third one could still be massless. A signal corresponding to a third mass difference was claimed by the LSND experiment (with antineutrinos) but not confirmed by KARMEN. More recently MiniBooNE ${ }^{3}$ has reported some possible supporting evidence for the LSND effect in their antineutrino run while no oscillation is observed in the neutrino run. The existence of a third oscillation frequency would imply the need for additional sterile neutrinos (i.e. with no weak interactions, as any new light active neutrino was excluded by LEP) or CPT violation (as, in this case, the masses of neutrinos and antineutrinos can be different).

The main recent developments on the experimental side were the results on $\theta_{13}$ from T2K ${ }^{4}$ and MINOS ${ }^{5}$ ) (very recently also DOUBLE CHOOZ ${ }^{6}$ ) ) and the coming back of sterile neutrinos. As well known, the T2K run was suddenly interrupted by the devastating earthquake that hit Japan on March 11, just minutes away from the scheduled presentation of the first $\mathrm{T} 2 \mathrm{~K}$ data. Later $\mathrm{T} 2 \mathrm{~K}$ released the first publication on their data ${ }^{4}$, reporting a $2.5 \sigma$ signal for $\sin ^{2} 2 \theta_{13}$ that indicates a value of $\theta_{13}$ close to the previous upper bound, of the order of the Cabibbo angle $\theta_{C}$.

On the evidence for sterile neutrinos a number of hints have been recently reported. They do not make yet a clear evidence but certainly pose an experimental problem that needs clarification. First, there is the MiniBooNE experiment 5 that in the antineutrino channel reports an excess of events supporting the LSND oscillation signal (originally observed with antineutrinos). More recently an update of the MiniBooNE data in the antineutrino channel shows less supporting evidence 7 . In the neutrino channel MiniBooNE did not observe a signal in the LSND domain. However, in these data there is a unexplained excess at low energy over the (reliably?) estimated background. In the neutrino data sample, for the search of a LSND-like signal, only the events with neutrino energy above a threshold value $E_{t h}$ were used, leaving the issue of an explanation of the low energy excess unanswered. In the antineutrino channel most of the support to the LSND signal appears to arise from an excess above $E_{t h}$ but quite close to it, so that there is, in my opinion, some room for perplexity. Then there is the reactor anomaly: a reevaluation of the reactor flux 8 ) produced an apparent gap between the theoretical expectations and the data taken at small distances from the reactor $(\lesssim 100 \mathrm{~m})$. The discrepancy is of the same order of the quoted systematic error whose estimate, detailed in the paper, should perhaps be reconsidered. Similarly the Gallium anomaly ${ }^{9}$ depends on the assumed cross-section which could be questioned. The reactor anomaly and the Gallium anomaly do not really agree on the oscillation parameters that they point to: the $\Delta m^{2}$ values are compatible but the central values of $\sin ^{2} 2 \theta$ differ by about an order of magnitude, with Gallium favouring the larger angle. Cosmological data allow the existence of one sterile neutrino, while the most stringent bounds arising form nucleosynthesis disfavour two or more sterile neutrinos 10 . Over all, only a small leakage from active 
to sterile neutrinos is allowed by present neutrino oscillation data, as discussed in refs. 11) If all the indications listed above were confirmed (it looks unlikely) then one sterile neutrino would not be enough and at least two would be needed with sub-eV masses. Establishing the existence of sterile neutrinos would be a great discovery. In fact a sterile neutrino is an exotic particle not predicted by the most popular models of new physics. A sterile neutrino is not a 4th generation neutrino: the latter is coupled to the weak interactions (it is active) and heavier than half the $\mathrm{Z}$ mass. A sterile neutrino would probably be a remnant of some hidden sector. The issue is very important so that new and better experimental data are badly needed.

As already mentioned, in neutrino oscillations the leakage from the three active species towards the sterile neutrinos is any case small and, in fact, the best established oscillation phenomena are well described in terms of 3-neutrino models. In this domain the main recent developments have been the T2K and MINOS results on $\theta_{13}$.

The T2K result ${ }^{4}$ ) , based on the observation of 6 electron events when $1.5 \pm 0.3$ are expected for $\theta_{13}=0$, is converted into a confidence interval $0.03(0.04) \leq \sin ^{2} 2 \theta_{13} \leq$ $0.28(0.34)$ at $90 \%$ c.l. for $\sin ^{2} 2 \theta_{23}=1,\left|\Delta m^{2}\right|=2.410^{-3} \mathrm{eV}^{2}, \delta_{C P}=0$ and for normal (inverted) neutrino mass hierarchy. Also the MINOS Collaboration released 5 ) their corresponding $90 \%$ c.l. range as $0(0) \leq \sin ^{2} 2 \theta_{13} \leq 0.12(0.19)$, which is displaced towards smaller values with respect to that of T2K. Finally DOUBLE CHOOZ $\underline{6}$ finds (with only the far detector in operation): $\sin ^{2} 2 \theta_{13}=0.085 \pm 0.051$ at $1 \sigma$.

\section{Neutrino Masses and Lepton Number Violation}

Neutrino oscillations imply non vanishing neutrino masses which in turn demand either the existence of right-handed $(\mathrm{RH})$ neutrinos (Dirac masses) or lepton number $\mathrm{L}$ violation (Majorana masses) or both. Given that neutrino masses are certainly extremely small, it is really difficult from the theory point of view to avoid the conclusion that $\mathrm{L}$ conservation must be violated. In fact, in terms of lepton number violation the smallness of neutrino masses can be explained as inversely proportional to the very large scale where $\mathrm{L}$ is violated, of order $M_{G U T}$ or even $M_{P l}$.

If $\mathrm{L}$ conservation is violated neutrinos can be Majorana fermions. For a Majorana neutrino each mass eigenstate with given helicity coincides with its own antiparticle with the same helicity. As well known, for a charged massive fermion there are four states differing by their charge and helicity (the four components of a Dirac spinor) as required by Lorentz and CPT invariance. For a massive Majorana neutrino, neutrinos and antineutrinos can be identified and only two components are needed to satisfy the Lorentz and CPT invariance constraints. Neutrinos can be Majorana fermions because, among the fundamental fermions (i.e. quarks and leptons), they are the only electrically neutral ones. If, and only if, the lepton number L is not conserved, i.e. it is not a good quantum number, then neutrinos and antineutrinos can be identified. For Majorana neutrinos both Dirac mass terms, that conserve $\mathrm{L}(\nu \rightarrow \nu)$, and Majorana 
mass terms, that violate L by two units $(\nu \rightarrow \bar{\nu})$, are in principle possible. Of course the restrictions from gauge invariance must be respected. So for neutrinos the Dirac mass terms $\left(\bar{\nu}_{R} \nu_{L}+\right.$ h.c. $)$ arise from the couplings with the Higgs field, as for all quarks and leptons. For Majorana masses, a $\nu_{L}^{T} \nu_{L}$ mass term has weak isospin 1 and needs two Higgs fields to make an invariant. On the contrary a $\nu_{R}^{T} \nu_{R}$ mass term is a gauge singlet and needs no Higgs. As a consequence, the right-handed neutrino Majorana mass $M_{R}$ is not bound to be of the order of the electroweak symmetry breaking (induced by the Higgs vacuum expectation value) and can be very large (see below).

Some notation: the charge conjugated of $\nu$ is $\nu^{c}$, given by $\nu^{c}=C(\bar{\nu})^{T}$, where $C=i \gamma_{2} \gamma_{0}$ is the charge conjugation matrix acting on the spinor indices (in the following, when dealing with the flavour structure of couplings, the $C$ matrix will be often omitted but understood). In particular $\left(\nu^{c}\right)_{L}=C\left(\overline{\nu_{R}}\right)^{T}$, so that, instead of using $\nu_{L}$ and $\nu_{R}$, we can refer to $\nu_{L}$ and $\left(\nu^{c}\right)_{L}$, or simply $\nu$ and $\nu^{c}$.

Once we accept $L$ non-conservation we gain an elegant explanation for the smallness of neutrino masses. If $\mathrm{L}$ is not conserved, even in the absence of heavy $\mathrm{RH}$ neutrinos, Majorana masses for neutrinos can be generated by dimension five operators 12 of the form

$$
O_{5}=\frac{(H l)_{i}^{T} \lambda_{i j}(H l)_{j}}{\Lambda}
$$

with $H$ being the ordinary Higgs doublet, $l_{i}$ the $\mathrm{SU}(2)$ lepton doublets, $\lambda$ a matrix in flavour space, $\Lambda$ a large scale of mass, of order $M_{G U T}$ or $M_{P l}$ and a charge conjugation matrix $C$ between the lepton fields is understood. Neutrino masses generated by $O_{5}$ are of the order $m_{\nu} \approx v^{2} / \Lambda$ for $\lambda_{i j} \approx \mathrm{O}(1)$, where $v \sim \mathrm{O}(100 \mathrm{GeV})$ is the vacuum expectation value of the ordinary Higgs.

We consider that the existence of $\mathrm{RH}$ neutrinos $\nu^{c}$ is quite plausible because most GUT groups larger than $\mathrm{SU}(5)$ require them. In particular the fact that $\nu^{c}$ completes the representation 16 of $\mathrm{SO}(10): 16=\overline{5}+10+1$, so that all fermions of each family are contained in a single representation of the unifying group, is too impressive not to be significant. At least as a classification group $\mathrm{SO}(10)$ must be of some relevance in a more fundamental layer of the theory! Thus in the following we both assume that $\nu^{c}$ exist and $\mathrm{L}$ is not conserved. With these assumptions the see-saw mechanism 13 is possible. We recall, also to fix notations, that in its simplest form it arises as follows. Consider the $\mathrm{SU}(3) \times \mathrm{SU}(2) \times \mathrm{U}(1)$ invariant Lagrangian giving rise to Dirac and $\nu^{c}$ Majorana masses (for the time being we consider the $\nu$ (versus $\nu^{c}$ ) Majorana mass terms as comparatively negligible):

$$
\mathcal{L}=-\nu^{c T} y_{\nu}(H l)+\frac{1}{2} \nu^{c T} M \nu^{c}+\text { h.c. }
$$

The Dirac mass matrix $m_{D} \equiv y_{\nu} v / \sqrt{2}$, originating from electroweak symmetry breaking, is, in general, non-hermitian and non-symmetric, while the Majorana mass matrix 
$M$ is symmetric, $M=M^{T}$. We expect the eigenvalues of $M$ to be of order $M_{G U T}$ or more because $\nu^{c}$ Majorana masses are $\mathrm{SU}(3) \times \mathrm{SU}(2) \times \mathrm{U}(1)$ invariant, hence unprotected and naturally of the order of the cutoff of the low-energy theory. Since all $\nu^{c}$ are very heavy we can integrate them away. For this purpose we write down the equations of motion for $\nu^{c}$ in the static limit, i.e. neglecting their kinetic terms:

$$
-\frac{\partial \mathcal{L}}{\partial \nu^{c}}=y_{\nu}(H l)-M \nu^{c}=0
$$

From this, by solving for $\nu^{c}$, we obtain:

$$
\nu^{c}=M^{-1} y_{\nu}(H l)
$$

We now replace in the lagrangian, eq. (2), this expression for $\nu^{c}$ and we get the operator $\mathrm{O}_{5}$ of eq. (1) with

$$
\frac{2 \lambda}{\Lambda}=-y_{\nu}^{T} M^{-1} y_{\nu}
$$

and the resulting neutrino mass matrix reads:

$$
m_{\nu}=m_{D}^{T} M^{-1} m_{D}
$$

This is the well known see-saw mechanism result 13): the light neutrino masses are quadratic in the Dirac masses and inversely proportional to the large Majorana mass. If some $\nu^{c}$ are massless or light they would not be integrated away but simply added to the light neutrinos. Notice that the above results hold true for any number $n$ of heavy neutral fermions $R$ coupled to the 3 known neutrinos. In this more general case $M$ is an $n$ by $n$ symmetric matrix and the coupling between heavy and light fields is described by the rectangular $n$ by 3 matrix $m_{D}$. Note that for $m_{\nu} \approx \sqrt{\Delta m_{a t m}^{2}} \approx 0.05$ $\mathrm{eV}$ (see Table(1) ) and $m_{\nu} \approx m_{D}^{2} / M$ with $m_{D} \approx v \approx 200 \mathrm{GeV}$ we find $M \approx 10^{15} \mathrm{GeV}$ which indeed is an impressive indication for $M_{G U T}$.

If additional non-renormalizable contributions to $\mathrm{O}_{5}$, eq. (1), are comparatively non-negligible, they should simply be added. For instance in $\mathrm{SO}(10)$ or in left-right extensions of the $\mathrm{SM}$, an $\mathrm{SU}(2)_{L}$ triplet can couple to two lepton doublets and to two Higgs and may induce a sizeable contribution to neutrino masses. At the level of the low-energy effective theory, such contribution is still described by the operator $O_{5}$ of eq. (1), obtained by integrating out the heavy $\mathrm{SU}(2)_{L}$ triplet. This contribution is called type II to be distinguished from that obtained by the exchange of RH neutrinos (type I). One can also have the exchange of a fermionic $\mathrm{SU}(2)_{L}$ triplet coupled to a lepton doublet and a Higgs (type III). After elimination of the heavy fields, at the level of the effective low-energy theory, the three types of see-saw terms are equivalent. In particular they have identical transformation properties under a chiral change of basis in flavour space. The difference is, however, that in type I see-saw 
mechanism, the Dirac matrix $m_{D}$ is presumably related to ordinary fermion masses because they are both generated by the Higgs mechanism and both must obey GUTinduced constraints. Thus more constraints are implied if one assumes the see-saw mechanism in its simplest type I version. .

\section{Basic Formulae and Data for Three-Neutrino Mixing}

We assume in the following that there are only two distinct neutrino oscillation frequencies, the atmospheric and the solar frequencies. These two can be reproduced with the known three light neutrino species (for more than three neutrinos see, for example, ref. 11 ).

Neutrino oscillations are due to a misalignment between the flavour basis, $\nu^{\prime} \equiv$ $\left(\nu_{e}, \nu_{\mu}, \nu_{\tau}\right)$, where $\nu_{e}$ is the partner of the mass and flavour eigenstate $e^{-}$in a lefthanded (LH) weak isospin $\mathrm{SU}(2)$ doublet (similarly for $\nu_{\mu}$ and $\nu_{\tau}$ ) and the mass eigenstates $\nu \equiv\left(\nu_{1}, \nu_{2}, \nu_{3}\right)$ 14|15):

$$
\nu^{\prime}=U \nu,
$$

where $U$ is the unitary 3 by 3 mixing matrix. Given the definition of $U$ and the transformation properties of the effective light neutrino mass matrix $m_{\nu}$ in eq. (1):

$$
\begin{aligned}
\nu^{\prime T} m_{\nu} \nu^{\prime} & =\nu^{T} U^{T} m_{\nu} U \nu \\
U^{T} m_{\nu} U & =\operatorname{Diag}\left(m_{1}, m_{2}, m_{3}\right) \equiv m_{\text {diag }},
\end{aligned}
$$

we obtain the general form of $m_{\nu}$ (i.e. of the light $\nu$ mass matrix in the basis where the charged lepton mass is a diagonal matrix):

$$
m_{\nu}=U^{*} m_{\text {diag }} U^{\dagger}
$$

The matrix $U$ can be parameterized in terms of three mixing angles $\theta_{12}, \theta_{23}$ and $\theta_{13}$ $\left(0 \leq \theta_{i j} \leq \pi / 2\right)$ and one phase $\varphi(0 \leq \varphi \leq 2 \pi)^{16}$, exactly as for the quark mixing matrix $V_{C K M}$. The following definition of mixing angles can be adopted:

$$
U=\left(\begin{array}{ccc}
1 & 0 & 0 \\
0 & c_{23} & s_{23} \\
0 & -s_{23} & c_{23}
\end{array}\right)\left(\begin{array}{ccc}
c_{13} & 0 & s_{13} e^{i \varphi} \\
0 & 1 & 0 \\
-s_{13} e^{-i \varphi} & 0 & c_{13}
\end{array}\right)\left(\begin{array}{ccc}
c_{12} & s_{12} & 0 \\
-s_{12} & c_{12} & 0 \\
0 & 0 & 1
\end{array}\right)
$$

where $s_{i j} \equiv \sin \theta_{i j}, c_{i j} \equiv \cos \theta_{i j}$. In addition, if $\nu$ are Majorana particles, we have the relative phases among the Majorana masses $m_{1}, m_{2}$ and $m_{3}$. If we choose $m_{3}$ real and positive, these phases are carried by $m_{1,2} \equiv\left|m_{1,2}\right| e^{i \phi_{1,2}}[17)$. Thus, in general, 9 parameters are added to the SM when non-vanishing neutrino masses are included: 3 eigenvalues, 3 mixing angles and $3 \mathrm{CP}$ violating phases.

In our notation the two frequencies, $\Delta m_{I}^{2} / 4 E$ ( $I=$ sun,atm), are parametrized in terms of the $\nu$ mass eigenvalues by

$$
\Delta m_{\text {sun }}^{2} \equiv\left|\Delta m_{12}^{2}\right|, \quad \Delta m_{\text {atm }}^{2} \equiv\left|\Delta m_{23}^{2}\right| \quad .
$$


where $\Delta m_{12}^{2}=\left|m_{2}\right|^{2}-\left|m_{1}\right|^{2}>0$ and $\Delta m_{23}^{2}=m_{3}^{2}-\left|m_{2}\right|^{2}$. The numbering $1,2,3$ corresponds to our definition of the frequencies and in principle may not coincide with the ordering from the lightest to the heaviest state. In fact, the sign of $\Delta m_{23}^{2}$ is not known and its determination is one of the existing experimental challenges. A positive (negative) sign corresponds to normal (inverse) hierarchy.

With the above definitions the present data are summarised in Table(1) 18), 19).

\begin{tabular}{|c|c|c|}
\hline Quantity & Fogli et al 18$)$ & Schwetz et al 19 \\
\hline$\Delta m_{\text {sun }}^{2}\left(10^{-5} \mathrm{eV}^{2}\right)$ & $7.58_{-0.26}^{+0.22}$ & $7.59_{-0.18}^{+0.20}$ \\
$\Delta m_{\text {atm }}^{2}\left(10^{-3} \mathrm{eV}^{2}\right)$ & $2.35_{-0.09}^{+0.12}$ & $2.50_{-0.16}^{+0.09}$ \\
$\sin ^{2} \theta_{12}$ & $0.312_{-0.016}^{+0.017}$ & $0.312_{-0.015}^{+0.017}$ \\
$\sin ^{2} \theta_{23}$ & $0.42_{-0.03}^{+0.08}$ & $0.52_{-0.07}^{+0.06}$ \\
$\sin ^{2} \theta_{13}$ & $0.025 \pm 0.007$ & $0.013_{-0.005}^{+0.007}$ \\
\hline
\end{tabular}

Table 1:

Fits to neutrino oscillation data. The results correspond to the new reactor fluxes. The fit of Schwetz et al ${ }^{19}$ refers to the normal hierarchy case (in the inverse hierarchy case the main difference is that $\left.\sin ^{2} \theta_{13}=0.016+0.008-0.006\right)$

Oscillation experiments do not provide information about the absolute neutrino mass scale. Limits on that are obtained ${ }^{1}$ from the endpoint of the tritium beta decay spectrum, from cosmology and from neutrinoless double beta decay $(0 \nu \beta \beta)$. From tritium we have an absolute upper limit of $2.2 \mathrm{eV}$ (at 95\% C.L.) 20 ) on the antineutrino mass eigenvalues involved in beta decay, which, combined with the observed oscillation frequencies under the assumption of three CPT-invariant light neutrinos, also amounts to an upper bound on the masses of the other active neutrinos. Complementary information on the sum of neutrino masses is also provided by the galaxy power spectrum combined with measurements of the cosmic microwave background anisotropies. According to recent analyses of the most reliable data 21) one obtains $\sum_{i}\left|m_{i}\right|<0.60 \div 0.75 \mathrm{eV}$ (at 95\% C.L.), depending on the retained data and the cosmological model priors assumed. These numbers for the sum have to be divided by 3 in order to obtain a limit on the mass of each light neutrino. The discovery of $0 \nu \beta \beta$ decay would be very important because it would establish lepton number violation and the Majorana nature of $\nu$ 's, and provide direct information on the absolute scale of neutrino masses. The present limit from $0 \nu \beta \beta$ (with large ambiguities from nuclear matrix elements) is about $\left|m_{e e}\right|<(0.3 \div 0.8) \mathrm{eV}^{22}$ (see eq. (13) $)$.

By now, after KamLAND, SNO and the upper limits on the absolute value of neutrino masses, not too much hierarchy in the spectrum of neutrinos is indicated by experiments 18), 19).

$$
r=\Delta m_{\text {sol }}^{2} / \Delta m_{\text {atm }}^{2}=0.032 \pm 0.002 \sim 1 / 30 .
$$


Thus, for a hierarchical spectrum, $m_{2} / m_{3} \sim \sqrt{r} \sim 0.2$, which is comparable to $\lambda_{C} \sim 0.226$ (thoughout this article $\lambda_{C}=\sin \theta_{C}$, with $\theta_{C}$ being the Cabibbo angle) or $\sqrt{m_{\mu} / m_{\tau}} \sim 0.24$. This suggests that the same hierarchy parameter (raised to powers with $\mathrm{O}(1)$ exponents) may apply for quark, charged lepton and neutrino mass matrices. In fact, $m_{\mu} / m_{\tau} \sim 0.06 \sim \lambda_{C}^{2}$ and $m_{e} / m_{\mu} \sim 0.005 \sim \lambda_{C}^{3-4}$ ).

For the near future the most important experimental challenges on neutrino oscillation experiments are more precise measurements of the absolute scale of neutrino mass (KATRIN, MARE), the accurate determination of $\theta_{13}$ (from MINOS, T2K and the reactor experiments DOUBLE CHOOZ, Daya Bay and RENO) and of the shift from maximal of $\theta_{23}$, the fixing of the sign of $\Delta m_{23}^{2}$ (normal or inverse hierarchy) (e.g. $\mathrm{NO} \nu \mathrm{A}$ ), the detection of $\mathrm{CP}$ violation in $\nu$ oscillations. Related to neutrino physics is the issue of the non conservation of the separate e, $\mu$ and $\tau$ lepton numbers. The recent new limit $\operatorname{Br}(\mu \rightarrow e \gamma) \lesssim 2.4 \cdot 10^{-12}$ obtained by the MEG experiment 23 is largely satisfied in the SM but it imposes a strong constraint on SUSY-GUT models.

\section{Importance of Neutrinoless Double Beta Decay}

The detection of neutrino-less double beta decay 24 would provide direct evidence of $L$ non conservation and of the Majorana nature of neutrinos. It would also offer a way to possibly disentangle the 3 cases of degenerate, normal or inverse hierachy neutrino spectrum. The quantity which is bound by experiments on $0 \nu \beta \beta$ is the 11 entry of the $\nu$ mass matrix, which in general, from $m_{\nu}=U^{*} m_{\text {diag }} U^{\dagger}$, is given by :

$$
\left|m_{e e}\right|=\left|\left(1-s_{13}^{2}\right)\left(m_{1} c_{12}^{2}+m_{2} s_{12}^{2}\right)+m_{3} e^{2 i \phi} s_{13}^{2}\right|
$$

where $m_{1,2}$ are complex masses (including Majorana phases) while $m_{3}$ can be taken as real and positive and $\phi$ is the $U_{P M N S}$ phase measurable from $\mathrm{CP}$ violation in oscillation experiments. Starting from this general formula it is simple to derive the bounds for degenerate, inverse hierarchy or normal hierarchy mass patterns shown in Fig. ${ }^{[25}$.

In the next few years a new generation of experiments (CUORE, GERDA, ....) will reach a larger sensitivity on $0 \nu \beta \beta$ by about an order of magnitude. Assuming the standard mechanism through mediation of a light massive Majorana neutrino, if these experiments will observe a signal this would indicate that the inverse hierarchy is realized, if not, then the normal hierarchy case remains a possibility.

\section{Baryogenesis via Leptogenesis from Heavy $\nu^{c}$ Decay}

In the Universe we observe an apparent excess of baryons over antibaryons. It is appealing that one can explain the observed baryon asymmetry by dynamical evolution (baryogenesis) starting from an initial state of the Universe with zero baryon number. For baryogenesis one needs the three famous Sakharov conditions: B violation, $\mathrm{CP}$ violation and no thermal equilibrium. In the history of the Universe these 


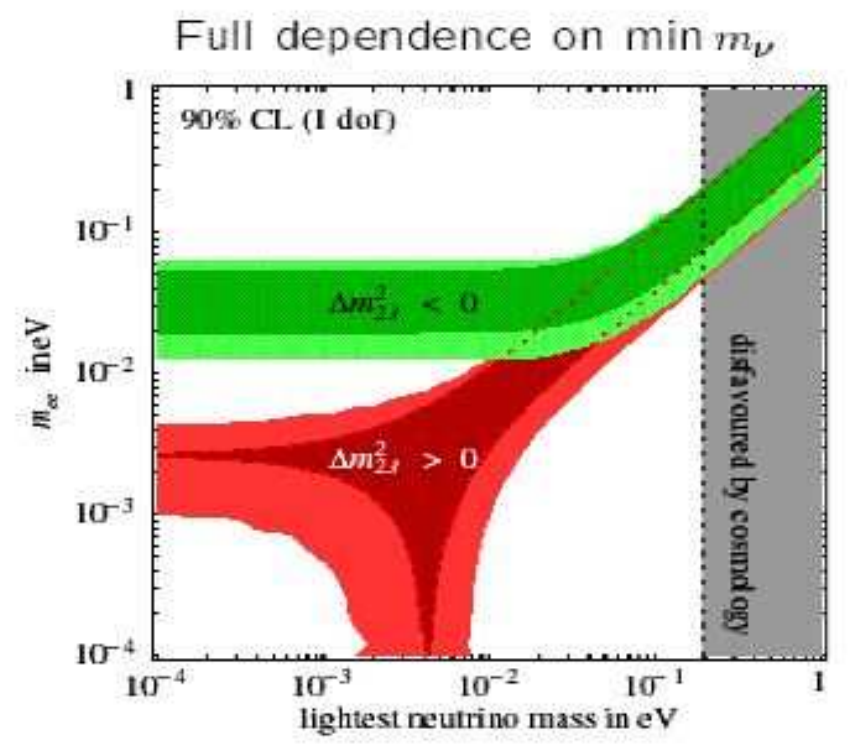

Figure 1: A plot 25 of $m_{e e}$ in $\mathrm{eV}$, the quantity measured in neutrino-less double beta decay, given in eq.(13), versus the lightest neutrino mass $m_{1}$, also in $\mathrm{eV}$. The upper (lower) band is for inverse (normal) hierarchy.

necessary requirements have possibly occurred at different epochs. Note however that the asymmetry generated during one such epoch could be erased in following epochs if not protected by some dynamical reason. In principle these conditions could be fulfilled in the SM at the electroweak phase transition. In fact, when kT is of the order of a few $\mathrm{TeV}, \mathrm{B}$ conservation is violated by instantons (but B-L is conserved), $\mathrm{CP}$ symmetry is violated by the CKM phase and sufficiently marked out-of- equilibrium conditions could be realized during the electroweak phase transition. So the conditions for baryogenesis at the weak scale in the SM superficially appear to be present. However, a more quantitative analysis 26 shows that baryogenesis is not possible in the $\mathrm{SM}$ because there is not enough $\mathrm{CP}$ violation and the phase transition is not sufficiently strong first order, unless the Higgs mass is below a bound which by now is excluded by LEP. In SUSY extensions of the SM, in particular in the MSSM, there are additional sources of CP violation and the bound on $m_{H}$ is modified but also this possibility has by now become at best marginal after the results from LEP2.

If baryogenesis at the weak scale is excluded by the data it can occur at or just below the GUT scale, after inflation. But only that part with $|\mathrm{B}-\mathrm{L}|>0$ would survive and not be erased at the weak scale by instanton effects. Thus baryogenesis at $k T \sim 10^{10}-10^{15} \mathrm{GeV}$ needs $\mathrm{B}-\mathrm{L}$ violation and this is also needed to allow $m_{\nu}$ if neutrinos are Majorana particles. The two effects could be related if baryogenesis arises from leptogenesis then converted into baryogenesis by instantons 27 ). The 


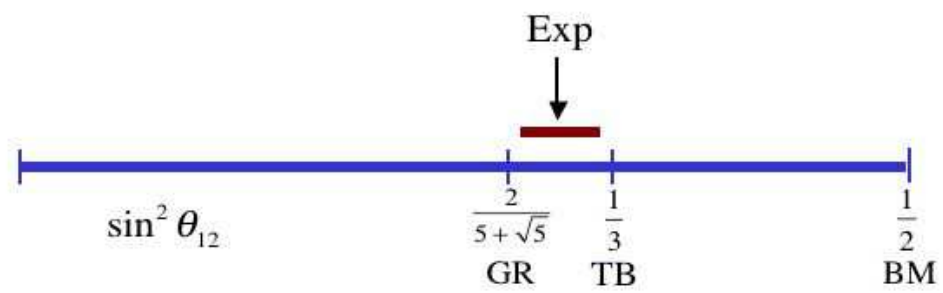

Figure 2: The values of $\sin ^{2} \theta_{12}$ for TB or GR or BM mixing are compared with the data

decays of heavy Majorana neutrinos (the heavy eigenstates of the see-saw mechanism) happen with violation of lepton number L, hence also of B-L and can well involve a sufficient amount of $\mathrm{CP}$ violation. Recent results on neutrino masses are compatible with this elegant possibility. Thus the case of baryogenesis through leptogenesis has been boosted by the recent results on neutrinos.

\section{Models of Neutrino Mixing}

Neutrino mixing is important because it could in principle provide new clues for the understanding of the flavour problem. Even more so since neutrino mixing angles show a pattern that is completely different than that of quark mixing: for quarks all mixing angles are small, for neutrinos two angles are large (one is even compatible with the maximal value) and only the third one is small. We first consider the case of models based on discrete flavour groups that have received a lot of attention in recent years 28 . There are a number of special mixing patterns that have been studied in this context. These mixing matrices all have $\sin ^{2} \theta_{23}=1 / 2, \sin ^{2} \theta_{13}=0$ and differ by the value of $\sin ^{2} \theta_{12}$ (see Fig. 2). The corresponding mass matrices are 2-3 symmetric, i.e. $\mu-\tau$ symmetric (see, as examples, the early work in ref.29] and the recent paper ref. $\left.{ }^{(30)}\right)$. The observed value of $\sin ^{2} \theta_{12}(18$, , 19) the best measured mixing angle, is very close, from below, to the so called Tri-Bimaximal (TB) value 31) which is $\sin ^{2} \theta_{12}=1 / 3$. Alternatively it is also very close, from above, to the Golden Ratio (GR) value 32 , , 33), 34) which is $\sin ^{2} \theta_{12}=\frac{1}{\sqrt{5} \phi}=\frac{2}{5+\sqrt{5}} \sim 0.276$, where $\phi=(1+\sqrt{5}) / 2$ is the GR (for a different connection to the GR in this context, see 35 , (36) $)$. Thus, a possibility is that one or the other of these coincidences is taken seriously and this leads to models where either TB or GR mixing is naturally predicted as a good first approximation. Here I will mainly refer to TB mixing which is the simplest and most studied first approximation to the data. 
The TB mixing matrix (in a particular phase convention) is given by:

$$
U_{T B}=\left(\begin{array}{ccc}
\sqrt{\frac{2}{3}} & \frac{1}{\sqrt{3}} & 0 \\
-\frac{1}{\sqrt{6}} & \frac{1}{\sqrt{3}} & -\frac{1}{\sqrt{2}} \\
-\frac{1}{\sqrt{6}} & \frac{1}{\sqrt{3}} & \frac{1}{\sqrt{2}}
\end{array}\right) .
$$

Note that the mixing angles are independent of mass ratios (while, for quark mixings, relations like $\lambda_{C}^{2} \sim m_{d} / m_{s}$ are typical). In the basis where charged lepton masses are diagonal, the effective neutrino mass matrix in the TB case is given by $m_{\nu}=U_{T B} \operatorname{diag}\left(\mathrm{m}_{1}, \mathrm{~m}_{2}, \mathrm{~m}_{3}\right) \mathrm{U}_{\mathrm{TB}}^{\mathrm{T}}$ :

$$
m_{\nu}=\left[\frac{m_{3}}{2} M_{3}+\frac{m_{2}}{3} M_{2}+\frac{m_{1}}{6} M_{1}\right] .
$$

where:

$$
M_{3}=\left(\begin{array}{ccc}
0 & 0 & 0 \\
0 & 1 & -1 \\
0 & -1 & 1
\end{array}\right), \quad M_{2}=\left(\begin{array}{ccc}
1 & 1 & 1 \\
1 & 1 & 1 \\
1 & 1 & 1
\end{array}\right), \quad M_{1}=\left(\begin{array}{ccc}
4 & -2 & -2 \\
-2 & 1 & 1 \\
-2 & 1 & 1
\end{array}\right) .
$$

The eigenvalues of $m_{\nu}$ are $m_{1}, m_{2}, m_{3}$ with eigenvectors $(-2,1,1) / \sqrt{6},(1,1,1) / \sqrt{3}$ and $(0,1,-1) / \sqrt{2}$, respectively. The expression in eq.(15) can be reproduced in models with sequential dominance or with form dominance, discussed by S. King and collaborators 37 .

As we see the most general neutrino mass matrix corresponding to TB mixing, in the basis of diagonal charged leptons, is of the form:

$$
m=\left(\begin{array}{ccc}
x & y & y \\
y & x+v & y-v \\
y & y-v & x+v
\end{array}\right)
$$

This is a symmetric, 2-3 symmetric matrix with $a_{11}+a_{12}=a_{22}+a_{23}$.

We now discuss models that naturally produce TB mixing in first approximation. Discrete non-abelian groups naturally emerge as suitable flavour symmetries 28, In fact the TB mixing matrix immediately suggests rotations by fixed, discrete angles. In a series of papers, started by 38 (for a rather complete list of references see 28), some recent papers are in ref.39) ) it has been pointed out that a broken flavour symmetry based on the discrete group $A_{4}$ appears to provide a simplest realization of this specific mixing pattern in Leading Order (LO). We recall that $A_{n}$ is the group of even permutations of $n$ objects ( $n ! / 2$ elements). In the case of GR mixing the simplest choice is the group $A_{5} 34$. Other solutions for TB mixing based on alternative discrete or continuous flavour groups have also been considered 28, (40) 
but the $A_{4}$ models have a very economical and attractive structure, e.g. in terms of group representations and of field content.

We recall that $A_{4}$ can be generated by the two elements $S$ and $T$ obeying the relations (a "presentation" of the group):

$$
S^{2}=(S T)^{3}=T^{3}=1
$$

The 12 elements of $A_{4}$ are obtained as: 1, S, T, ST, TS, $T^{2}, S T^{2}, S T S, T S T, T^{2} S$, $T S T^{2}, T^{2} S T$. The inequivalent irreducible representations of $A_{4}$ are 1,1 ', 1 "' and 3. Note that the squares of the dimensions of all these representations add up to 12 , the dimension of $A_{4}$. It is immediate to see that one-dimensional unitary representations are given by:

$$
\begin{array}{lll}
1 & S=1 & T=1 \\
1^{\prime} & S=1 & T=e^{i 4 \pi / 3} \equiv \omega^{2} \\
1^{\prime \prime} & S=1 & T=e^{i 2 \pi / 3} \equiv \omega
\end{array}
$$

The three-dimensional unitary representation, in a basis where the element $T$ is diagonal, is given by:

$$
T=\left(\begin{array}{ccc}
1 & 0 & 0 \\
0 & \omega^{2} & 0 \\
0 & 0 & \omega
\end{array}\right), \quad S=\frac{1}{3}\left(\begin{array}{ccc}
-1 & 2 & 2 \\
2 & -1 & 2 \\
2 & 2 & -1
\end{array}\right)
$$

Note that the generic mass matrix for TB mixing in eq.(17) can be specified as the most general matrix that is invariant under $\mu-\tau$ symmetry, implemented by the unitary matrix $A_{\mu \tau}$ :

$$
A_{\mu \tau}=\left(\begin{array}{lll}
1 & 0 & 0 \\
0 & 0 & 1 \\
0 & 1 & 0
\end{array}\right)
$$

and under the $S$ transformation:

$$
m=S m S, \quad m=A_{\mu \tau} m A_{\mu \tau}
$$

where $\mathrm{S}$ is given in eq.(20).

The $m$ mass matrix of eq.(17) is derived in the basis where charged leptons are diagonal. It is useful to consider the product $m^{2}=m_{e}^{\dagger} m_{e}$, where $m_{e}$ is the charged lepton mass matrix (defined as $\bar{\psi}_{R} m_{e} \psi_{L}$ ), because this product transforms as $m^{\prime 2}=$ $U_{e}^{\dagger} m^{2} U_{e}$, with $U_{e}$ the unitary matrix that rotates the left-handed (LH) charged lepton fields. The most general diagonal $\mathrm{m}^{2}$ is invariant under a diagonal phase matrix with 3 different phase factors:

$$
m_{e}^{\dagger} m_{e}=T^{\dagger} m_{e}^{\dagger} m_{e} T
$$

and conversely a matrix $m_{e}^{\dagger} m_{e}$ satisfying the above requirement is diagonal. If $T^{n}=$ 1 the matrix $T$ generates a cyclic group $Z_{n}$. The simplest case is $n=3$, which corresponds to $Z_{3}$ (but $n>3$ is equally possible) and to the $T$ matrix in eq.(20). 
We can now see why $A_{4}$ works for TB mixing. It works because $S$ and $T$ are matrices of $A_{4}$ (in fact they satisfy eqs.(18)). One could object that the matrix $A_{23}$ is not an element of $A_{4}$ (because the 2-3 exchange is an odd permutation). But it can be shown that in $A_{4}$ models the 2-3 symmetry is maintained by imposing that there are no flavons transforming as $1^{\prime}$ or $1^{\prime \prime}$ that break $A_{4}$ with two different VEV's: in particular one can assume that there are no flavons in the model transforming as $1^{\prime}$ or $1^{\prime \prime} 41$.

The group $A_{4}$ has two obvious subgroups: $G_{S}$, which is a reflection subgroup generated by $S$ and $G_{T}$, which is the group generated by $T$, which is isomorphic to $Z_{3}$. If the flavour symmetry associated to $A_{4}$ is broken by the VEV of a triplet $\varphi=\left(\varphi_{1}, \varphi_{2}, \varphi_{3}\right)$ of scalar fields, there are two interesting breaking pattern. The VEV

$$
\langle\varphi\rangle=\left(v_{S}, v_{S}, v_{S}\right)
$$

breaks $A_{4}$ down to $G_{S}$, while

$$
\langle\varphi\rangle=\left(v_{T}, 0,0\right)
$$

breaks $A_{4}$ down to $G_{T}$. We have seen that $G_{S}$ and $G_{T}$ are the relevant low-energy symmetries of the neutrino and the charged-lepton sectors, respectively. Indeed we have shown that the TB mass matrix is invariant under $G_{S}$ and, for charged leptons, a diagonal $m_{e}^{\dagger} m_{e}$ is invariant under $G_{T}$. A crucial part of all serious A4 models is the dynamical generation of this alignment in a natural way. In most of the models $A_{4}$ is accompanied by additional flavour symmetries, either discrete like $Z_{N}$ or continuous like $\mathrm{U}(1)$, which are necessary to eliminate unwanted couplings, to ensure the needed vacuum alignment (obtained from the minimization of the most general potential compatible with the assumed symmetries) and to reproduce the observed mass hierarchies. Explicit realizations of models for TB mixing based on $A_{4}$ can be found, for example, in 41/42/43/44/45). The possible origin of $A_{4}$ from a deeper level of the theory has been discussed in the context of extra dimensions and orbifolding 43 , 46) or as related to the fact that $A_{4}$ is a subgroup of the modular group ${ }^{42}$, which plays a role in string theory. In passing, we note that we are mainly interested here in the possibility that the flavour symmetry is valid at the GUT scale and is broken at lower scales.

\section{Applying $A_{4}$ to lepton masses and mixings}

In the lepton sector a typical $A_{4}$ model works as follows 42 . One assigns leptons to the four inequivalent representations of $A_{4}$ : LH lepton doublets $l$ transform as a triplet 3 , while the $\mathrm{RH}$ charged leptons $e^{c}, \mu^{c}$ and $\tau^{c}$ transform as $1,1^{\prime \prime}$ and $1^{\prime}$, respectively. Here we consider a see-saw realization, so we also introduce conjugate neutrino fields $\nu^{c}$ transforming as a triplet of $A_{4}$. The fact that LH lepton doublets $l$ and, in the see-saw case, also the RH neutrinos $\nu^{c}$, transform as triplets is crucial to realize the fixed ratios of mass matrix elements needed to obtain TB mixing. A 
drawback is that for the ratio $r$, defined in eq.(12), one would expect $\sqrt{r} \approx \mathcal{O}(1)$ to be compared with the experimental value is $\sqrt{r} \approx 0.2$, which implies a moderate fine tuning.

One adopts a supersymmetric (SUSY) context also to make contact with Grand Unification (flavor symmetries are supposed to act near the GUT scale). In fact, as well known, SUSY is important in GUT's for offering a solution to the hierarchy problem, for improving coupling unification and for making the theory compatible with bounds on proton decay. But, in models of lepton mixing, SUSY also helps for obtaining the vacuum alignment, because the SUSY constraints are very strong and limit the form of the superpotential very much. Thus SUSY is not necessary but it is a plausible and useful ingredient. The flavor symmetry is broken by two triplets $\varphi_{S}$ and $\varphi_{T}$ (with the vacuum alignment in eqs.(24, 25)) and by one or more singlets $\xi$. All these fields are invariant under the SM gauge symmetry. Two Higgs doublets $h_{u, d}$, invariant under $A_{4}$, are also introduced. One can obtain the observed hierarchy among $m_{e}, m_{\mu}$ and $m_{\tau}$ by introducing an additional $\mathrm{U}(1)_{F N}$ flavor symmetry 47) under which only the RH lepton sector is charged (recently some models were proposed with a different VEV alignment such that the charged lepton hierarchies are obtained without introducing a $U(1)$ symmetry 48|45) $)$. We recall that $U(1)_{F N}$ is a simplest flavor symmetry where particles in different generations are assigned (in general) different values of an Abelian charge. Also Higgs fields may get a non zero charge. When the symmetry is spontaneously broken the entries of mass matrices are suppressed if there is a charge mismatch and more so if the corresponding mismatch is larger. We assign FN-charges $0, q$ and $2 q$ to $\tau^{c}, \mu^{c}$ and $e^{c}$, respectively. There is some freedom in the choice of $q$. Here we take $q=2$. By assuming that a flavon $\theta$, carrying a negative unit of $\mathrm{FN}$ charge, acquires a $\operatorname{VEV}\langle\theta\rangle / \Lambda \equiv \lambda<1$, the Yukawa couplings become field dependent quantities $y_{e, \mu, \tau}=y_{e, \mu, \tau}(\theta)$ and we have

$$
y_{\tau} \approx \mathcal{O}(1) \quad, \quad y_{\mu} \approx O\left(\lambda^{2}\right), \quad y_{e} \approx O\left(\lambda^{4}\right)
$$

Had we chosen $q=1$, we would have needed $\langle\theta\rangle / \Lambda$ of order $\lambda^{2}$, to reproduce the above result. The superpotential term for lepton masses, $w_{l}$ is given by:

$w_{l}=y_{e} e^{c}\left(\varphi_{T} l\right)+y_{\mu} \mu^{c}\left(\varphi_{T} l\right)^{\prime}+y_{\tau} \tau^{c}\left(\varphi_{T} l\right)^{\prime \prime}+y\left(\nu^{c} l\right)+\left(x_{A} \xi+\tilde{x}_{A} \tilde{\xi}\right)\left(\nu^{c} \nu^{c}\right)+x_{B}\left(\varphi_{S} \nu^{c} \nu^{c}\right)+\ldots$

with dots denoting higher dimensional operators that lead to corrections to the LO approximation. In our notation, the product of 2 triplets (33) transforms as 1, (33)' transforms as $1^{\prime}$ and $(33)^{\prime \prime}$ transforms as $1^{\prime \prime}$. To keep our formulae compact, we omit to write the Higgs and flavon fields $h_{u, d}, \theta$ and the cut-off scale $\Lambda$. For instance $y_{e} e^{c}\left(\varphi_{T} l\right)$ stands for $y_{e} e^{c}\left(\varphi_{T} l\right) h_{d} \theta^{4} / \Lambda^{5}$. The parameters of the superpotential $w_{l}$ are complex, in particular those responsible for the heavy neutrino Majorana masses, $x_{A, B}$. Some terms allowed by the $A_{4}$ symmetry, such as the terms obtained by the exchange $\varphi_{T} \leftrightarrow \varphi_{S}$, (or the term $\left(\nu^{c} \nu^{c}\right)$ ) are missing in $w_{l}$. Their absence is crucial and, in each version of $A_{4}$ models, is motivated by additional symmetries. 
As for the neutrino spectrum both normal and inverted hierarchies can be realized. It is interesting that $A_{4}$ models with the see-saw mechanism typically lead to a light neutrino spectrum which satisfies the sum rule (among complex masses):

$$
\frac{1}{m_{3}}=\frac{1}{m_{1}}-\frac{2}{m_{2}}
$$

A detailed discussion of a spectrum of this type can be found in refs. 42/45). The above sum rule gives rise to bounds on the lightest neutrino mass. As a consequence, for example, the possible values of $\left|m_{e e}\right|$ are restricted. For normal hierarchy we have

$$
\left|m_{e e}\right| \approx \frac{4}{3 \sqrt{3}} \Delta m_{\text {sun }}^{2} \approx 0.007 \mathrm{eV} .
$$

while for inverted hierarchy

$$
\left|m_{e e}\right| \geq \sqrt{\frac{\Delta m_{a t m}^{2}}{8}} \approx 0.017 \mathrm{eV} .
$$

In a completely general framework, without the restrictions imposed by the flavor symmetry, $\left|m_{e e}\right|$ could vanish in the case of normal hierarchy. In this model $\left|m_{e e}\right|$ is always different from zero, though its value for normal hierarchy is probably too small to be detected in the next generation of $0 \nu \beta \beta$ experiments.

In the leading approximation $A_{4}$ models lead to exact TB mixing. In these models TB mixing is implied by the symmetry at the leading order approximation which is corrected by non leading effects. Given the set of flavour symmetries and having specified the field content, the non leading corrections to TB mixing, arising from higher dimensional effective operators, can be evaluated in a well defined expansion. In the absence of specific dynamical tricks, in a generic model, all the three mixing angles receive corrections of the same order of magnitude. Since the experimentally allowed departures of $\theta_{12}$ from the TB value, $\sin ^{2} \theta_{12}=1 / 3$, are small, numerically not larger than $\mathcal{O}\left(\lambda_{C}^{2}\right)$, it follows that both $\theta_{13}$ and the deviation of $\theta_{23}$ from the maximal value are expected to also be typically of the same general size. The central values $\sin \theta_{13} \sim 0.16-0.11$ that can be derived from the experimental results in the two columns of Table(1), respectively, are in between $\mathcal{O}\left(\lambda_{C}^{2}\right) \sim \mathcal{O}(0.05)$ and $\mathcal{O}\left(\lambda_{C}\right) \sim \mathcal{O}(0.23)$. Thus models based on TB or GR mixing are still viable with preference for the lower side of the experimental range for $\theta_{13}$. It is also to be noted that one can introduce some additional theoretical input to enhance the value of $\theta_{13}$. In the case of $A_{4}$, examples are provided by the model of ref. ${ }^{49}$, formulated before the T2K and MINOS results were known and the modified $A_{4}$ model of ref.50) (see also $(51)$.

\section{8. $A_{4}$, quarks and GUT's}

Much attention has been devoted to the question whether models with TB mixing in the neutrino sector can be suitably extended to also successfully describe the 
observed pattern of quark mixings and masses and whether this more complete framework can be made compatible with (supersymmetric) SU(5) or SO(10) Grand Unification.

The simplest attempts of directly extending models based on $A_{4}$ to quarks have not been satisfactory. At first sight the most appealing possibility is to adopt for quarks the same classification scheme under $A_{4}$ that one has used for leptons (see, for example, ref.$\sqrt[42]{2})$. Thus one tentatively assumes that LH quark doublets $Q$ transform as a triplet 3 , while the antiquarks $\left(u^{c}, d^{c}\right),\left(c^{c}, s^{c}\right)$ and $\left(t^{c}, b^{c}\right)$ transform as $1,1^{\prime \prime}$ and $1^{\prime}$, respectively. This leads to $V_{u}=V_{d}$ and to the identity matrix for $V_{C K M}=V_{u}^{\dagger} V_{d}$ in the lowest approximation. This at first appears as very promising: a LO approximation where neutrino mixing is $\mathrm{TB}$ and $V_{C K M}=1$ is a very good starting point. But there are some problems. First, the corrections to $V_{C K M}=1$ turn out to be strongly constrained by the leptonic sector, because lepton mixing angles are very close to the TB values, and, in the simplest models, this constraint leads to a too small $V_{u s}$ (i.e. the Cabibbo angle is rather large in comparison to the allowed shifts from the TB mixing angles). Also in these models, the quark classification which leads to $V_{C K M}=1$ is not compatible with $A_{4}$ commuting with $\mathrm{SU}(5)$. An additional consequence of the above assignment is that the top quark mass arises from a non-renormalizable dimension- 5 operator. In that case, to reproduce the top mass, we need to compensate the cutoff suppression by some extra dynamical mechanism. Alternatively, we have to introduce a separate symmetry breaking parameter for the quark sector, sufficiently close to the cutoff scale.

Due to this, larger discrete groups have been considered for the description of quarks. A particularly appealing set of models is based on the discrete group $T^{\prime}$, the double covering group of $A_{4}$ [52), 53), 54). The representations of $T^{\prime}$ are those of $A_{4}$ plus three independent doublets $2,2^{\prime}$ and $2^{\prime \prime}$. The doublets are interesting for the classification of the first two generations of quarks ${ }^{55}$. For example, in ref. 53 , a viable description was obtained, i.e. in the leptonic sector the predictions of the $A_{4}$ model are maintained, while the $T^{\prime}$ symmetry plays an essential role for reproducing the pattern of quark mixing. But, again, the classification adopted in this model is not compatible with Grand Unification.

As a result, the group $A_{4}$ was considered by many authors to be too limited to also describe quarks and to lead to a grand unified description. But it has been shown 44) that this negative attitude is not justified and that it is actually possible to construct a viable model based on $A_{4}$ which leads to a grand unified theory (GUT) of quarks and leptons with TB mixing for leptons and with quark (and charged lepton) masses and mixings compatible with experiment. At the same time this model offers an example of an extra dimensional SU(5) GUT in which a description of all fermion masses and mixings is accomplished. The formulation of $\mathrm{SU}(5)$ in extra dimensions has the usual advantages of avoiding large Higgs representations to break SU(5) and of solving the doublet-triplet splitting problem. The choice of the transformation 
properties of the two Higgses $H_{5}$ and $H_{\overline{5}}$ has a special role in this model. They are chosen to transform as two different $A_{4}$ singlets 1 and $1^{\prime}$. As a consequence, mass terms for the Higgs colour triplets are not directly allowed and their masses are introduced by orbifolding, à la Kawamura [56). In this model, proton decay is dominated by gauge vector boson exchange giving rise to dimension- 6 operators, while the usual contribution of dimension- 5 operators is forbidden by the selection rules of the model. Given the large $M_{G U T}$ scale of SUSY models and the relatively huge theoretical uncertainties, the decay rate is within the present experimental limits. A see-saw realization in terms of an $A_{4}$ triplet of $\mathrm{RH}$ neutrinos $\nu^{c}$ ensures the correct ratio of light neutrino masses with respect to the GUT scale. In this model extra dimensional effects directly contribute to determine the flavour pattern, in that the two lightest tenplets $T_{1}$ and $T_{2}$ are in the bulk (with a doubling $T_{i}$ and $T_{i}^{\prime}, i=1,2$ to ensure the correct zero mode spectrum), whereas the pentaplets $F$ and $T_{3}$ are on the brane. The hierarchy of quark and charged lepton masses and of quark mixings is determined by a combination of extra dimensional suppression factors and of $U(1)_{F N}$ charges, both of which only apply to the first two generations, while the neutrino mixing angles derive from $A_{4}$ in the usual way. If the extra dimensional suppression factors and the $U(1)_{F N}$ charges are switched off, only the third generation masses of quarks and charged leptons survive. Thus the charged fermion mass matrices are nearly empty in this limit (not much of $A_{4}$ effects remain) and the quark mixing angles are determined by the small corrections induced by the above effects. The model is natural, since most of the small parameters in the observed pattern of masses and mixings as well as the necessary vacuum alignment are justified by the symmetries of the model. However, in this case, like in all models based on $U(1)_{F N}$, the number of $\mathcal{O}(1)$ parameters is larger than the number of measurable quantities, so that in the quark sector the model can only account for the orders of magnitude (measured in terms of powers of an expansion parameter) and not for the exact values of mass ratios and mixing angles. A moderate fine tuning is only needed to enhance the Cabibbo mixing angle between the first two generations, which would generically be of $\mathcal{O}\left(\lambda_{C}^{2}\right)$.

The problem of constructing GUT models based on $S U(5) \otimes G_{f}$ or $S O(10) \otimes G_{f}$ with approximate TB mixing in the leptonic sector has also been considered by many authors. Examples are: for $G_{f}=A_{4}$ ref. $\left[57\right.$, for $T^{\prime}$ ref. $\stackrel{54}{ }$, for $S_{4}$ ref. ${ }^{58}$. As for the models based on $S O(10) \otimes G_{f}$ recent examples were discussed with $G_{f}=S_{4}$ and $G_{f}=P S L_{2}(7)$ 60) . Clearly the case of $S O(10)$ is even more difficult than that of $S U(5)$ because the neutrino sector is tightly related to that of quarks and charged leptons as all belong to the 16 of $S O(10)$. For a discussion of $S O(10) \otimes A_{4}$ models, see 61). More in general see refs $\underline{62}$. In our opinion most of the models are incomplete (for example, the crucial issue of VEV alignment is not really treated in depth as it should) and/or involve a number of unjustified steps and ad-hoc fine tuning of parameters.

While $A_{4}$ is the minimal flavor group leading to TB mixing, alternative flavor 
groups have been studied in the literature and can lead to interesting variants with some specific features. Actually, in ref $[63$, the claim was made that, in order to obtain the TB mixing "without fine tuning", the finite group must be $S_{4}$ or a larger group containing $S_{4}$. For us this claim is not well grounded being based on an abstract mathematical criterium for a natural model (see also 64 ). For us a physical field theory model is natural if the interesting results are obtained from the most general lagrangian compatible with the stated symmetry and the specified representation content for the flavons. For example, we obtain from $A_{4}$ (which is a subgroup of $S_{4}$ ) a natural (in our sense) model for the TB mixing by simply not including symmetry breaking flavons transforming like the $1^{\prime}$ and the $1^{\prime \prime}$ representations of $A_{4}$. This limitation on the transformation properties of the flavons is not allowed by the rules specified in ref.63) which demand that the symmetry breaking is induced by all possible kinds of flavons (note that, according to this criterium, the SM of electroweak interactions would not be natural because only Higgs doublets are introduced!). Rather, for naturalness we also require that additional physical properties like the VEV alignment or the hierarchy of charged lepton masses also follow from the assumed symmetry and are not obtained by fine tuning parameters: for this actually $A_{4}$ can be more effective than $S_{4}$ because it possesses three different singlet representations $1,1^{\prime}$ and $1^{\prime \prime}$.

Models of neutrino mixing based on $S_{4}$ have in fact been studied 65). The group of the permutations of 4 objects $S_{4}$ has 24 elements and 5 equivalence classes that correspond to 5 inequivalent irreducible representations, two singlets, one doublet, two triplets: $1_{1}, 1_{2}, 2,3_{1}$ and $3_{2}$. Note that the squares of the dimensions of all these representations add up to 24 .

\section{Bimaximal Mixing and S4}

The new results showing that probably $\theta_{13}$ is not far from its former upper bound could alternatively be interpreted as an indication that the agreement with the TB or GR mixing is accidental. Then a scheme where instead the Bimaximal (BM) mixing is the correct first approximation modified by terms of $\mathcal{O}\left(\lambda_{C}\right)$ could be relevant. In BM mixing $\theta_{12}$ and $\theta_{23}$ are both maximal while $\theta_{13}=0$ (see. Fig. 2). This is in line with the well known empirical observation that $\theta_{12}+\theta_{C} \sim \pi / 4$, a relation known as quark-lepton complementarity $\left[66\right.$, or similarly $\theta_{12}+\sqrt{m_{\mu} / m_{\tau}} \sim \pi / 4$. No compelling model leading, without parameter fixing, to the exact complementarity relation has been produced so far. Probably the exact complementarity relation becomes more plausible if replaced with $\theta_{12}+\mathcal{O}\left(\theta_{C}\right) \sim \pi / 4$ or $\theta_{12}+\mathcal{O}\left(m_{\mu} / m_{\tau}\right) \sim \pi / 4$ (which we could call "weak" complementarity). One can think of models where, because of a suitable symmetry, BM mixing holds in the neutrino sector at leading order and the necessary, rather large, corrective terms for $\theta_{12}$ arise from the diagonalization of charged lepton masses 66 . These terms of order $\mathcal{O}\left(\lambda_{C}\right)$ from the charged lepton 
sector would then generically also affect $\theta_{13}$ and the resulting value could well be compatible with the present experimental values of $\theta_{13}$. A word of caution must be kept in mind: in the presence of these relatively large off diagonal terms in the charged lepton diagonalizing matrix one must arrange that not too large contributions to the decays $\mu \rightarrow e \gamma$ or $\tau \rightarrow \mu \gamma$ are generated ${ }^{28}$.

The BM mixing matrix is given by:

$$
U_{B M}=\left(\begin{array}{ccc}
\frac{1}{\sqrt{2}} & -\frac{1}{\sqrt{2}} & 0 \\
\frac{1}{2} & \frac{1}{2} & -\frac{1}{\sqrt{2}} \\
\frac{1}{2} & \frac{1}{2} & \frac{1}{\sqrt{2}}
\end{array}\right) .
$$

Along this line of thought, we have used the expertise acquired with non Abelian finite flavour groups to construct a model ${ }^{67}$ ) based on the permutation group $S_{4}$ which naturally leads to the BM mixing at leading order. We have adopted a supersymmetric formulation of the model in 4 space-time dimensions. The complete flavour group is $S_{4} \times Z_{4} \times U(1)_{F N}$. In leading order, the charged leptons are diagonal and hierarchical and the light neutrino mass matrix, after see-saw, leads to the exact BM mixing. The model is built in such a way that the dominant corrections to the BM mixing, from higher dimensional operators in the superpotential, only arise from the charged lepton sector at next-to-the-leading-order and naturally inherit $\lambda_{C}$ (which fixes the charged lepton mass hierarchies) as the relevant expansion parameter. As a result the mixing angles deviate from the BM values by terms of $\mathcal{O}\left(\lambda_{C}\right)$ (at most), and weak complementarity holds. A crucial feature of the model is that only $\theta_{12}$ and $\theta_{13}$ are corrected by terms of $\mathcal{O}\left(\lambda_{C}\right)$ while $\theta_{23}$ is unchanged at this order (which is essential for a better agreement of the model with the present data). Recently the model was extended to include quarks in a $S U(5)$ Grand Unified version 68 or in a Pati-Salam framework ${ }^{69}$. An $S O(10)$ model is discussed in ref.70).

\section{Anarchy versus Symmetry}

We now briefly turn to models that do not take seriously any of the coincidences described above (i.e. the proximity of the data to the TB or GR patterns or the quarklepton complementarity: these patterns cannot all be true and it is possible that none of them is true) and are therefore based on a less restrictive flavour symmetry. It is clear that the $\mathrm{T} 2 \mathrm{~K}$ hint that $\theta_{13}$ may be large is great news for the most extreme position of this type, which is "anarchy" 71): no symmetry at all in the lepton sector, only chance. This view predicts generic mixing angles, so the largest angle, $\theta_{23}$, should somewhat deviate from maximal and the smallest angle, $\theta_{13}$, should be as large as possible within the experimental bounds. Anarchy can be formulated in a

$S U(5) \otimes U(1)$ context by taking different Froggatt-Nielsen charges 47) only for the 
$S U(5)$ tenplets (for example 10: $(3,2,0)$ where 3 is the charge of the first generation, 2 of the second, zero of the third) while no charge differences appear in the $\overline{5}: \overline{5}:(0,0,0)$. This assignment is in agreement with the empirical fact that the mass hierarchies are more pronounced for up quarks in comparison with down quarks and charged leptons. In a non see-saw model, with neutrino masses dominated by the contribution of the dimension-5 Weinberg operator in eq.(1), the $\overline{5}$ vanishing charges directly lead to random neutrino mass and mixing matrices. In anarchical see-saw models also the charges of the $\mathrm{SU}(5)$ singlet $\mathrm{RH}$ neutrinos must be undifferentiated among the 3 generations: 1: $(0,0,0)$. Anarchy can be mitigated by assuming that it only holds in the 2-3 sector: e.g $\overline{5}$ : $(2,0,0)$ with the advantage that the first generation masses and the angle $\theta_{13}$ are then naturally small (see ref. ${ }^{72}$ ) for a recent discussion of this model). In models with see-saw one can alternatively play with the charges for the RH SU(5) singlet neutrinos. If, for example, we take $1:(1,-1,0)$, together with $\overline{5}$ : $(2,0,0)$, it is possible to get a normal hierarchy model with $\theta_{13}$ small and also with

$r=\Delta m_{\text {solar }}^{2} / \Delta m_{\text {atm }}^{2}$ naturally small (see, for example, ref. ${ }^{73}$ ). In summary anarchy and its variants, all based on chance, offer a rather economical class of models that are among those that are compatible with the recent $\theta_{13}$ results, with preference with the upper side of the experimentally allowed range.

\section{Conclusion}

In the last decade we have learnt a lot about neutrino masses and mixings. A list of important conclusions have been reached. Neutrinos are not all massless but their masses are very small. Probably masses are small because neutrinos are Majorana particles with masses inversely proportional to the large scale $\mathrm{M}$ of lepton number violation. It is quite remarkable that $\mathrm{M}$ is empirically not far from $M_{G U T}$, so that neutrino masses fit well in the SUSY GUT picture. Also out of equilibrium decays with $\mathrm{CP}$ and $\mathrm{L}$ violation of heavy $\mathrm{RH}$ neutrinos can produce a B-L asymmetry, then converted near the weak scale by instantons into an amount of B asymmetry compatible with observations (baryogenesis via leptogenesis) 27). It has been established that most probably active neutrinos are not a significant component of dark matter in the Universe. We have also understood there there is no contradiction between large neutrino mixings and small quark mixings, even in the context of GUTs.

This is a very impressive list of achievements. Coming to a detailed analysis of neutrino masses and mixings a long collection of models have been formulated over the years. With continuous improvements of the data and more precise values of the mixing angles most of the models have been discarded by experiment. Still the surviving models span a wide range going from a maximum of symmetry, with discrete non-abelian flavour groups, to the opposite extreme of anarchy. By now, besides the detailed knowledge of the entries of the $V_{C K M}$ matrix we also have a reasonable determination of the neutrino mixing matrix $U_{P M N S}$. The data appear 
to suggest some special patterns (recall Fig. 2) like TB or GR or BM mixing to be valid in some leading approximation, corrected by small non leading terms. If one takes these "coincidences" seriously, then non-abelian discrete flavour groups emerge as the main road to an understanding of this mixing pattern. Indeed the entries of e.g. TB mixing matrix are clearly suggestive of "rotations" by simple, very specific angles. It is remarkable that neutrino and quark mixings have such a different qualitative pattern. An obvious question is whether some additional indication for discrete flavour groups can be obtained by considering the extension of the models to the quark sector, perhaps in a Grand Unified context. The answer appears to be that, while the quark masses and mixings can indeed be reproduced in models where TB or BM mixing is realized in the leptonic sector through the action of discrete groups, there are no specific additional hints in favour of discrete groups that come from the quark sector. Further important input could come from $\mu \rightarrow e \gamma$ and in general from lepton flavour violating processes, from $b \rightarrow s \gamma$ and from LHC physics. In fact, new physics at the weak scale could have important feedback on the physics of neutrino masses and mixing.

In conclusion, one could have imagined that neutrinos would bring a decisive boost towards the formulation of a comprehensive understanding of fermion masses and mixings. In reality it is frustrating that no real illumination was sparked on the problem of flavour. We can reproduce in many different ways the observations, in a wide range that goes from anarchy to discrete flavour symmetries) but we have not yet been able to single out a unique and convincing baseline for the understanding of fermion masses and mixings. In spite of many interesting ideas and the formulation of many elegant models the mysteries of the flavour structure of the three generations of fermions have not been much unveiled.

I imagine that by the next edition of this by now classic School, we will know the value of $\theta_{13}$ with a better accuracy, from the continuation of T2K, MINOS and DOUBLE CHOOZ and from the start of Daya Bay and RENO. Some existing models will be eliminated and the surviving ones will be updated to become more quantitative in order to cope with a precisely known mixing matrix. A definitely non vanishing $\theta_{13}$ value will encourage the planning of long baseline experiments for the detection of $\mathrm{CP}$ violation in neutrino oscillations. Along the way the important issue of the existence of sterile neutrinos must be clarified. The on going or in preparation experiments on the absolute value of neutrino masses, on $0 \nu \beta \beta$, on $\mu \rightarrow e \gamma$, on the search for dark matter etc can also lead to extremely important developments in the near future. So this field is very promising and there are all reasons to expect an exciting time ahead of us.

\section{Acknowledgments}

I warmly thank Prof. Antonino Zichichi for his kind invitation and for the impres- 
sive hospitality received in Erice, as usual. I also thank the Staff of the E. Majorana School, in particular Mrs. Fiorella Ruggiu for their assistance in organizing and managing my stay at the School. I am indebted with Luca Merlo for his critical reading of a preliminary version of this work. I am also glad to acknowledge interesting discussions on this subject with him and with Ferruccio Feruglio and Davide Meloni. Supported in part by PRIN 2008 and by the EU network LHCPHENONET.

\section{References}

1) G. Altarelli and F. Feruglio, New J. Phys. $6 \quad$ (2004) 106 [ArXiv:hep-ph/0405048]; G. Altarelli, arXiv:1011.5342.

2) R. N. Mohapatra and A. Y. Smirnov, Ann. Rev. Nucl. Part. Sci. 56, 569 (2006) ArXiv:hep-ph/0603118; W. Grimus, PoS P2GC:001,2006. ArXiv:hep-ph/0612311]; M. C. Gonzalez-Garcia and M. Maltoni, Phys. Rept. 460 (2008) 1 [arXiv:0704.1800].

3) The MiniBooNE Collaboration Phys.Rev.Lett. 105181801 (2010), arXiv:1007.1150.

4) T2K Collaboration: K.Abe et al, Phys. Rev. Lett. 107 (2011) 041801, arXiv:1106.2822.

5) MINOS Collaboration, P. Adamson et al, arXiv:1108.0015.

6) DOUBLE CHOOZ Collaboration, H. de Kerret, Talk at LowNu, Seoul, November 2011.

7) Z. Djurcic, Talk at NuFact2011, Geneva, August 2011.

8) G. Mention et al, Phys.Rev.D83:073006,2011, [arXiv:1101.2755]; Th. A. Mueller et al, Phys.Rev.C83:054615,2011, arXiv:1101.2663; P. Huber, Phys.Rev.C84:024617,2011, arXiv:1106.0687] .

9) P. Anselmann et al. (GALLEX), Phys. Lett. B342, 440 (1995); W. Hampel et al. (GALLEX), Phys. Lett. B420, 114 (1998); F. Kaether et al. Phys. Lett. B685, 47 (2010); J. N. Abdurashitov et al. (SAGE), Phys. Rev. Lett. 77, 4708 (1996); J. N. Abdurashitov et al. (SAGE), Phys. Rev. C59, 2246 (1999); J. N. Abdurashitov et al., Phys. Rev. C73, 045805 (2006); J. N. Abdurashitov et al. (SAGE), Phys. Rev. C80, 015807 (2009).

10) E. Giusarma et al, arXiv:1102.4774

11) C. Giunti, arXiv:1110.3914 and references therein.

12) S. Weinberg, Phys.Rev.Lett. 431566 (1979).

13) P. Minkowski, Phys. Letters B67 (1977)421; T. Yanagida, in Proc. of the Workshop on Unified Theory and Baryon Number in the Universe, KEK, March 1979; S. L. Glashow, in "Quarks and Leptons", Cargèse, ed. M. Lévy et al., Plenum, 1980 New York, p. 707; M. Gell-Mann, P. Ramond and R. Slansky, in Supergravity, Stony Brook, Sept 1979; R. N. Mohapatra and G. Senjanovic, Phys. Rev. Lett. 44 (1980) 912. 
14) B. Pontecorvo, Sov. Phys. JETP 6, 429 (1957) [Zh. Eksp. Teor. Fiz. 33, 549 (1957)]; Z. Maki, M. Nakagawa and S. Sakata, Prog. Theor. Phys. 28 (1962) 870; B. Pontecorvo, Sov. Phys. JETP 26, 984 (1968) [Zh. Eksp. Teor. Fiz. 53, 1717 (1968)]; V. N. Gribov and B. Pontecorvo, Phys. Lett. B 28, 493 (1969).

15) B. W. Lee, S. Pakvasa, R. Shrock, and H. Sugawara, Phys. Rev. Lett. 38, 937 (1977); B. W. Lee and R. Shrock, Phys. Rev. D16, 1444 (1977).

16) N. Cabibbo, Phys. Lett. B 72, 333 (1978).

17) S. M. Bilenky, J. Hosek and S. T. Petcov, Phys. Lett. B 94 (1980) 495; J. Schechter and J. W. F. Valle, Phys. Rev. D 22 (1980) 2227; M. Doi, T. Kotani, H. Nishiura, K. Okuda and E. Takasugi, Phys. Lett. B 102 (1981) 323. Mass matrices with a general dependence on $\phi, \phi_{1,2}$ have been analyzed in: M. Frigerio and A. Y. Smirnov, Nucl. Phys. B 640 (2002) 233 and Phys. Rev. D 67 (2003) 013007.

18) G. L. Fogli et al, arXiv:1106.6028.

19) T. Schwetz, M. Tortola and J. W. F. Valle, arXiv:1108.1376].

20) Ch. Weinheimer et al, Phys. Lett. B460 (1999) 219.

21) G. L. Fogli et al, arXiv:0805.2517, E. Komatsu, et al., Astrophys.J.Suppl.192:18,2011, arXiv:1001.4538], M. C. Gonzalez-Garcia, M. Maltoni and J. Salvado, JHEP 1008:117,2010, arXiv:1006.3795].

22) W. Rodejohann, Int.J.Mod.Phys. E20, 1833(2011), arXiv:1106.1334.

23) T. Mori, Talk at the EPS Conference, Grenoble, July 2011.

24), A. S. Barabash, Phys. Atom. Nucl. 73 (2010) 162.

25) F. Feruglio, A. Strumia and F Vissani, Nucl.Phys.B637:345-377,2002., Addendum-ibid.B659:359-362, 2003. [ArXiv:hep-ph/0201291].

26) For a review see, for example,: M. Trodden, Rev. Mod. Phys. 71, 1463 (1999).

27) For a review see for example: W. Buchmuller, R.D. Peccei and T. Yanagida, Ann.Rev.Nucl.Part.Sci.55:311-355,2005. ArXiv:hep-ph/0502169].

28) G. Altarelli and F. Feruglio, Rev.Mod.Phys. 82 (2010) 2701, arXiv:1002.0211.

29) T. Fukuyama and H. Nishiura, [ArXiv:hep-ph/9702253].

30) H.-J. He and F.-R. Yin, Phys.Rev. D84 (2011) 033009, arXiv:1104.2654.

31) P. F. Harrison, D. H. Perkins and W. G. Scott, Phys. Lett. B 530 (2002) 167, ArXiv:hep-ph/0202074; P. F. Harrison and W. G. Scott, Phys. Lett. B 535 (2002) 163, [ArXiv:hep-ph/0203209]; Phys. Lett. B 547 (2002) 219, [ArXiv:hep-ph/0210197]; Phys. Lett. B 557 (2003) 76, ArXiv:hep-ph/0302025]; Z. z. Xing, Phys. Lett. B 533 (2002) 85, ArXiv:hep-ph/0204049.

32) Y. Kajiyama, M. Raidal and A. Strumia, Phys. Rev. D 76 (2007) 117301 arXiv:0705.4559.

33) L. L. Everett and A. J. Stuart, Phys.Rev.D79:085005,2009, [arXiv:0812.1057]

34) F. Feruglio and A. Paris, JHEP 1103 (2011) 101, arXiv:1101.0393. 
35) W. Rodejohann, Phys. Lett. B671 (2009) 267, arXiv:0810.5239 [hep-ph]].

36) A. Adulpravitchai, A. Blum and W. Rodejohann, New J. Phys. 11 (2009) 063026 arXiv:0903.0531].

37) S. F. King, arXiv:1106.4239 and references therein.

38) E. Ma and G. Rajasekaran, Phys. Rev. D 64 (2001) 113012 ArXiv:hep-ph/0106291]; E. Ma, Mod. Phys. Lett. A 17 (2002) 627 ArXiv:hep-ph/0203238. K. S. Babu, E. Ma and J. W. F. Valle, Phys. Lett. B 552 (2003) 207 [ArXiv:hep-ph/0206292]; M. Hirsch, J. C. Romao, S. Skadhauge, J. W. F. Valle and A. Villanova del Moral, [ArXiv:hep-ph/0312244], Phys. Rev. D 69 (2004) 093006 [ArXiv:hep-ph/0312265]; E. Ma, Phys. Rev. D 70 (2004) 031901; Phys. Rev. D 70 (2004) 031901 [ArXiv:hep-ph/0404199]; New J. Phys. 6 (2004) 104 ArXiv:hep-ph/0405152; ArXiv:hep-ph/0409075; S. L. Chen, M. Frigerio and E. Ma, Nucl. Phys. B 724 (2005) 423 ArXiv:hep-ph/0504181]; E. Ma, Phys. Rev. D 72 (2005) 037301 |ArXiv:hep-ph/0505209|.

39) F. del Aguila, A. Carmona and J. Santiago, [ArXiv:1001.515]; A. Kadosh and E. Pallante, arXiv:1004.0321]; D. Meloni, S. Morisi and E. Peinado, arXiv:1011.1371; E. Peinado, arXiv:1010.2614]; F. Feruglio and A. Paris, Nucl.Phys. B840 (2010) 405, arXiv:1005.5526.

40) H. Ishimori et al, Prog.Theor.Phys.Suppl.183:1, 2010; arXiv:1003.3552; P. O. Ludl, J.Phys.A A43 (2010) 395204, arXiv:1006.1479; W. Grimus and P.O. Ludl, J.Phys.A43 (2010) 445209 [arXiv:1006.0098]; K. M. Parattu and A. Wingerter, Phys.Rev.D84:013011,2011, arXiv:1012.2842].

41) G. Altarelli and F. Feruglio, Nucl. Phys. B $\mathbf{7 2 0}$ (2005) 64 ArXiv:hep-ph/0504165].

42) G. Altarelli and F. Feruglio, Nucl. Phys. B $\mathbf{7 4 1}$ (2006) 215 ArXiv:hep-ph/0512103.

43) G. Altarelli, F. Feruglio and Y. Lin, Nucl. Phys. B 775 (2007) 31 ArXiv:hep-ph/0610165].

44) G. Altarelli, F. Feruglio and C. Hagedorn, JHEP 0803 (2008) 052 arXiv:0802.0090].

45) G. Altarelli and D. Meloni, J.Phys.G G36 (2009) 085005, arXiv:0905.0620].

46) A. Adulpravitchai, A. Blum and M. Lindner, JHEP 07 (2009) 053 arXiv:0906.0468]; A. Adulpravitchai, M.A. Schmidt, arXiv:1001.3172]; T.J. Burrows, S.F. King, Nucl.Phys. B842 (2011) 107, arXiv:1007.2310.

47) C. D. Froggatt and H. B. Nielsen, Nucl. Phys. B147 (1979) 277.

48) Y. Lin, Nucl. Phys. B 813, 91 (2009) arXiv:0804.2867]; [arXiv:0903.0831];

49) Y. Lin, Nucl.Phys. B824 (2010) 95, arXiv:0905.3534.

50) E. Ma and D. Wegman, [arXiv:1106.4269].

51) I. de Medeiros Varzielas and L. Merlo, JHEP 1102 (2011) 062, 
arXiv:1011.6662.

52) P.H. Frampton and T.W. Kephart, Int. J. Mod. Phys. A10 (1995) 4689, ArXiv:hep-ph/9409330; JHEP 09 (2007) 110, arXiv:0706.1186; A. Aranda, C.D. Carone and R.F. Lebed, Phys. Lett. B474 (2000) 170, ArXiv:hep-ph/9910392; Phys. Rev. D62 (2000) 016009, ArXiv:hep-ph/0002044]; P.D. Carr and P.H. Frampton, ArXiv:hep-ph/0701034]; A. Aranda, Phys. Rev. D76 (2007) 111301, arXiv:0707.3661]; P.H. Frampton and S. Matsuzaki, Phys. Lett. B679 (2009) 347, arXiv:0902.1140]; G.J. Ding, Phys. Rev. D78 (2008) 036011, arXiv:0803.2278;

53) F. Feruglio et al, Nucl. Phys. B775 (2007) 120, ArXiv:hep-ph/0702194].

54) M.C. Chen and K.T. Mahanthappa, Phys. Lett. B652 (2007) 34, arXiv:0705.0714.

55) A. Pomarol and D. Tommasini, Nucl. Phys. B466 ( 1996) 3, ArXiv:hep-ph/9507462; R. Barbieri, G.R. Dvali and L. Hall, Phys. Lett. B377 (1996) 76, [ArXiv:hep-ph/9512388] R. Barbieri et al, Nucl. Phys. B493 (1997) 3, ArXiv:hep-ph/9610449]; R. Barbieri, L. Hall and A. Romanino, Phys. Lett. B401 (1997) 47, [ArXiv:hep-ph/9702315].

56) Y. Kawamura, Prog. Theor. Phys. 105 (2001) 999, [ArXiv:hep-ph/0012125].

57) E. Ma, Mod. Phys. Lett. A 20 (2005) 2767, ArXiv:hep-ph/0506036;

E. Ma, H. Sawanaka and M. Tanimoto, Phys. Lett. B 641 (2006) 301 ArXiv:hep-ph/0606103]; E. Ma, Mod. Phys. Lett. A 21 (2006) 2931 ArXiv:hep-ph/0607190]; S. Morisi, M. Picariello and E. Torrente-Lujan, Phys. Rev. D 75 (2007) 075015 [ArXiv:hep-ph/0702034]; W. Grimus and H. Kuhbock, arXiv:0710.1585; P. Ciafaloni et al, Phys. Rev. D79 (2009) 116010, arXiv:0901.2236]; F. Bazzocchi et al, J. Phys. G36 (2009) 015002, arXiv:0802.1693]; S. Antusch, S.F. King and M. Spinrath, arXiv:1005.0708.

58) C. Hagedorn, S.F. King and C. Luhn, arXiv:1003.4249]; H. Ishimoriet al, arXiv:1004.5004.

59) B. Dutta, Y. Mimura and R.N. Mohapatra, Phys. Rev. D80 (2009) 095021, arXiv:0910.1043]; JHEP 05 (2010) 034, arXiv:0911.2242];

60) S. F. King and C. Luhn, arXiv:0912.1344.

61) F. Bazzocchi, F. Frigerio and S. Morisi, Phys. Rev. D78 (2008)116018, arXiv:0809.3573.

62) G. Altarelli and G. Blankenburg, JHEP 1103 (2011) 133, arXiv:1012.2697; A.S. Joshipura, K.M. Patel, JHEP09(2011)137, arXiv:1105.5943; Phys.Rev.D83:095002,2011, arXiv:1102.5148; P. S. Bhupal Dev, R. N. Mohapatra and M. Severson, arXiv:1107.2378.

63) C. S. Lam, Phys.Rev.Lett.101:121602,2008, arXiv:0804.2622]; Phys. Rev. D 78 (2008) 073015, arXiv:0809.1185].

64) W. Grimus, L. Lavoura and P. O. Ludl, J.Phys. G36 (2009) 15007, 
arXiv:0906.2689].

65) R.N. Mohapatra, M.K. Parida and G. Rajasekaran, Phys. Rev. D69 (2004) 053007, ArXiv:hep-ph/0301234]; C. Hagedorn, M. Lindner and R.N. Mohapatra, JHEP 06 (2006) 042, [ArXiv:hep-ph/0602244]; Y. Cai and H.B Yu, Phys. Rev. D74 (2006) 115005, ArXiv:hep-ph/0608022]; E. Ma, Phys. Lett. B632 (2006) 352; ArXiv:hep-ph/0508231]; F. Bazzocchi and S. Morisi, Phys. Rev. D80 (2009) 096005; arXiv:0811.0345]; H. Ishimori, Y. Shimizu and M. Tanimoto, Prog. Theor. Phys. 121 (2009) 769, arXiv:0812.5031; F. Bazzocchi, L. Merlo and S. Morisi, Nucl. Phys B816 ( 2009 ) 204, arXiv:0901.2086; Phys. Rev. D80 (2009) 053003, arXiv:0902.2849; D. Meloni, J. Phys. G37 (2010) 055201,[ArXiv: 0911.3591]. G.J. Ding, Nucl. Phys. B827 (2010) 82, arXiv:0909.2210; S. Morisi and E. Peinado, Phys. Rev. D81 (2010) 085015, arXiv:1001.2265;

66) M. Raidal, Phys. Rev. Lett. 93 (2004) 16180, ArXiv:hep-ph/0404046]; H. Minakata and A. Y. Smirnov, Phys. Rev. D 70 (2004) 073009, ArXiv:hep-ph/0405088; $\quad$ H. Minakata, ArXiv:hep-ph/0505262; P. H. Frampton and R. N. Mohapatra, JHEP 0501, 025 (2005), ArXiv:hep-ph/0407139;J. Ferrandis and S. Pakvasa, Phys. Rev. D 71 (2005) 033004, ArXiv:hep-ph/0412038; S. K. Kang, C. S. Kim and J. Lee, ArXiv:hep-ph/0501029]; G. Altarelli, F. Feruglio and I. Masina, Nucl. Phys. B 689 (2004) 157 ArXiv:hep-ph/0402155]; N. Li and B. Q. Ma, ArXiv:hep-ph/0501226; K. Cheung, et al, ArXiv:hep-ph/0503122; Z. z. Xing, [ArXiv:hep-ph/0503200]; A. Datta, L. Everett and P. Ramond, [ArXiv:hep-ph/0503222]; T. Ohlsson, [ArXiv:hep-ph/0506094]; S. Antusch, S. F. King and R. N. Mohapatra, [ArXiv:hep-ph/0504007]; M. Lindner, M. A. Schmidt and A. Y. Smirnov, [ArXiv:hep-ph/0505067]; S. F. King, JHEP 0508 (2005) 105 [ArXiv:hep-ph/0506297]; A. Dighe, S. Goswami, and P. Roy, Phys.Rev.D73 (2006) 07130, |ArXiv:hep-ph/0602062]; B. C. Chauhanet al, Eur.Phys. J.C50(2007) 573, |ArXiv:hep-ph/0605032 M. A. Schmidt and A. Yu. Smirnov, Phys.Rev. D74(2006)113003, [ArXiv:hep-ph/0607232];

K. A Hochmuth and W. Rodejohann, Phys.Rev. D75(2007) 073001, ArXiv:hep-ph/0607103; F. Plentinger, G. Seidl and W. Winter, Nucl.Phys. B791 (2008) 60, [ArXiv: hep-ph/0612169]; Phys.Rev. D76 (2007)113003, arXiv:0707.2379; Y. H. Ahn, H-Y. Cheng and S. Oh, Phys.Rev.D83:076012,2011, arXiv:1102.0879].

67) G. Altarelli, F. Feruglio and L. Merlo, JHEP 0905 (2009) 020, arXiv:0903.1940].

68) D. Meloni, JHEP 1110 (2011) 010, arXiv:1107.0221.

69) R. de Adelhart Toorop., F. Bazzocchi and L. Merlo, JHEP 1008 (2010) 001, arXiv:1003.4502].

70) K. M. Patel, Phys.Lett.B695:225, 2011 arXiv:1008.5061. 
71) L. J. Hall, H. Murayama and N. Weiner, Phys.Rev.Lett. 84 (2000) 2572, ArXiv:hep-ph/9911341].

72) W. Buchmuller, V. Domcke, and K. Schmitz, arXiv:1111.3872].

73) G. Altarelli, F. Feruglio and I. Masina, JHEP 0301 (2003) 035, ArXiv:hep-ph/0210342. 